\title{
ARTIFICIAL INTELLIGENCE SYSTEMS IN MICROMECHANICS
}

\author{
Felipe Lara-Rosano, Ernst Kussul, Tatiana Baidyk, Leopoldo Ruiz, Alberto \\ Caballero, Graciela Velasco \\ CCADET, UNAM
}

\begin{abstract}
Some of the artificial intelligence (AI) methods could be used to improve the automation system performance in manufacturing processes. However, the implementation of these AI methods in the industry is rather slow, because of the high cost of the experiments with the conventional manufacturing and AI systems. To lower the experiment cost in this field, we have developed a special micromechanical equipment, similar to conventional mechanical equipment, but of much smaller size and therefore of lower cost. This equipment could be used for evaluation of different AI methods in an easy and inexpensive way. The proved methods could be transferred to the industry through appropriate scaling. In this paper we describe the prototypes of low cost microequipment for manufacturing processes and some AI method implementations to increase its precision, like computer vision systems based on neural networks for microdevice assembly, and genetic algorithms for microequipment characterization and microequipment precision increase.
\end{abstract}

Key words: artificial intelligence, micromechanics, computer vision, genetic algorithms

\section{INTRODUCTION}

The development of AI technologies opens an opportunity to use them not only for conventional applications (expert systems, intelligent data bases [1], technical diagnostics [2,3] etc.), but also for total automation of mechanical manufacturing. Such AI methods as adaptive critic design [4,5], adaptive fuzzy Petri networks [6,7], neural network based computer vision systems [8-12], etc. could be used to solve the automation problems. To check this opportunity up, it is necessary to create an experimental factory 
with fully automated manufacturing processes. This is a very difficult and expensive task.

\section{MICROEQUIPMENT TECHNOLOGY}

To make a very small mechanical microequipment, a new technology was proposed $[13,14]$. This technology is based on micromachine tools and microassembly devices, which can be produced as sequential generations of microequipment. Each generation should include equipment (machine-tools, manipulators, assembly devices, measuring instruments, etc.) sufficient for manufacturing an identical equipment set of smaller size. Each subsequent equipment generation could be produced by the preceding one. The equipment size of each subsequent generation is smaller than the overall size of preceding generation.

The first-generation microequipment can be produced by conventional large-scale equipment. Using microequipment of this first generation, a second microequipment generation having smaller overall sizes can be produced.

We call this approach to mechanical microdevices manufacturing MicroEquipment Technology (MET) [15].

The proposed MET technology has many advantages:

(1) The equipment miniaturization leads to decreasing the occupied space as well as energy consumption, and, therefore, the cost of the products.

(2) The labor costs are bound to decrease due to the reduction of maintenance costs and a higher level of automation expected in MET.

(3) Miniaturization of equipment by MET results in a decrease of its cost. This is a consequence of the fact that microequipment itself becomes the object of MET. The realization of universal microequipment that is capable of extended reproduction of itself will allow the manufacture of low-cost microequipment in a few reproductive acts because of the lower consumption of materials, energy, labor, and space in MET. Thus the miniaturization of equipment opens the way to a drastic decrease in the unit cost of individual processing.

At a lower unit cost of individual micromachining, the most natural way to achieve high throughput is to parallelize the processes of individual machining by concurrent use of a great quantity of microequipment of the same kind. Exploitation of that great number of microsized machine-tools is only feasible with their automatic operation and a highly automated control of the microfactory as a whole. We expect that many useful and proved concepts, ideas and techniques of automation can be borrowed from mechanical engineering. They vary from the principles of factory automation 
(FMS and CAM) to the ideas of unified containers and clamping devices and techniques of numerical control. However automation of micromanufacturing has peculiarities that will require the special methods of artificial intelligence.

\section{AI BASED CONTROL SYSTEM FOR MICROMECHANICAL FACTORY}

Let us consider a general hierarchical structure of the automatic control system for a micromechanical factory. The lowest (first) level of the system controls the micromechanical equipment (the micro machine-tools and assembly manipulators), provides the simplest microequipment diagnostics and the final measurement and testing of production. The second level of the control system controls the devices that transport workpieces, tools, parts, and the whole equipment items; coordinates the operation of the lowest level devices; provides the intermediate quality inspection of production and the more advanced diagnostics of equipment condition. The third control level contains the system for the automatic choice of process modes and routes for parts machining. The top (fourth) level of the control system performs detecting of non-standard and alarm situations and decision making, including communication with the operator.

We proceed from the assumption that no more than one operator will manage the microfactory. It means that almost all the problems arising at any control level during the production process should be solved automatically and that operator must solve only a few problems, that are too complex or unusual to be solved automatically.

Since any production process is affected by various disturbances, the control system should be an adaptive one. Moreover, it should be selflearning, because it is impossible to foresee all kinds of disturbances in advance. AI that is able to construct the self-learning algorithms and to minimize the participation of operator, seem to be especially useful for this task. AI includes different methods for creating autonomous control systems. The neural classifiers will be particularly useful at the lowest level of the control system. They could be used for the selection of treatment modes, checking of cutting tool conditions, control of the assembly processes, etc. They allow to make the control system more flexible. The system will automatically compensate for small deviations of production conditions, such as the change of cutting tool shape or external environment parameters, variations in the structure of workpiece materials, etc. AI will permit to design self-learning classifiers and should provide the opportunity to exclude the participation of human operator at this level of control. 
At the second control level, the AI system should detect all deviations from the normal production process and make decisions about how to modify the process to compensate for the deviation. The compensation should be made by tuning the parameters of the lower level control systems. The examples of such deviations are the deviations from the production schedule, failures in some devices, off-standard production, etc. At this level the AI system should contain the structures in which the interrelations of production process constituents are represented. As in the previous case, it is desirable to have the algorithms working without the supervisor.

The third control level is connected basically with the change of nomenclature or volume of the production manufactured by the factory. It is convenient to develop such a system so that the set-up costs for a new production or the costs to change the production volume should be minimal. The self-learning AI structures formed at the lowest level could provide the basis for such changes of set-up by selection of the process parameters, the choice of equipment configuration for machining and assembly, etc. At the third control level the AI structures should detect the similarity of new products with the products which were manufactured in the past. On the basis of this similarity, the proposals about the manufacturing schedule, process modes, routing, etc. will be automatically formed. Then they will be checked up by the usual computational methods of computer aided manufacturing (CAM). The results of the check, as well as the subsequent information about the efficiency of decisions made at this level, may be used for improving the AI system.

The most complicated AI structures should be applied at the top control level. This AI system level must have the ability to reveal the recent unusual features in the production process, to make the evaluation of possible influence of these new features on the production process, and to make decisions for changing the control system parameters at the various hierarchical levels or for calling for the operator's help. At this level, the control system should contain the intelligence knowledge base, which can be created using the results of the operation of the lower level control systems and the expert knowledge. At the beginning, the expert knowledge of macromechanics may be used.

At present many methods of AI are successfully used in the industry $[16,17]$. They could be used also for micromechanics. But the problems of fully automated microfactory creation can not be investigated experimentally in conventional industry because of the high cost of the experiments. Here we propose to develop low cost micromechanical test bed to solve these problems.

The prototypes of the first generation microequipment are designed and examined in the Laboratory of Micromechanics and Mechatronics, 
CCADET, UNAM. The prototypes use adaptive algorithms of the lowest level. At present more sophisticated algorithms based on neural networks and genetic algorithms are being developed. Below we describe our experiments in the area of such algorithms development and applications.

\section{DEVELOPMENT OF MICROEQUIPMENT PROTOTYPES AND ADAPTIVE ALGORITHMS}

\subsection{Micromachine Tools}

The developed prototype of the first generation micromachine tool is shown in Fig. 1. We have been exploiting this prototype for approximately four years for experimental work and student training.

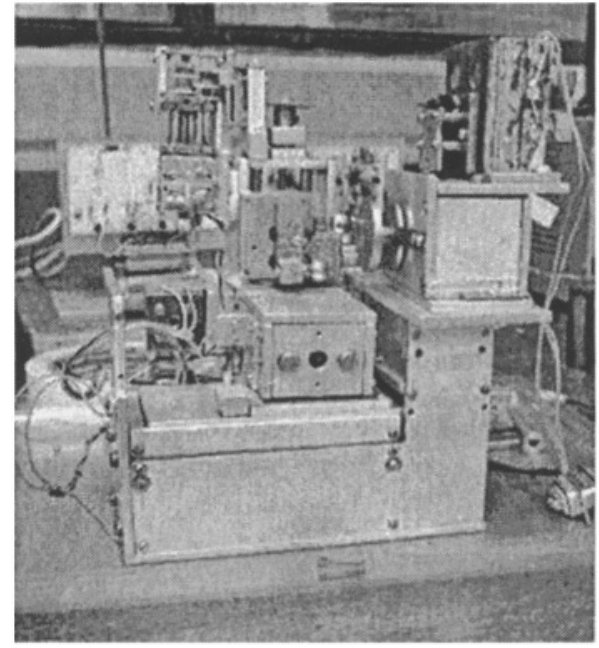

Figure 1. The developed second prototype of the first generation of micromachine tool.

This prototype of the micromachine tool has the size $130 \times 160 \times 85 \mathrm{~mm}^{3}$ and is controlled by a PC. The axes $X$ and $Z$ have $20 \mathrm{~mm}$ of displacement and the $Y$-axis has $35 \mathrm{~mm}$ of displacement; all have the same configuration. The resolution is $1.87 \mu \mathrm{m}$ per motor step.

\subsection{Micromanipulators}

At present, in the Laboratory of Micromechanics and Mechatronics, CCADET, UNAM the principles, designs and methods of manufacture of micromachine tools and micromanipulators corresponding to the first 
microequipment generation are developed. All these works are accompanied with of the prototypes development (Fig.2).

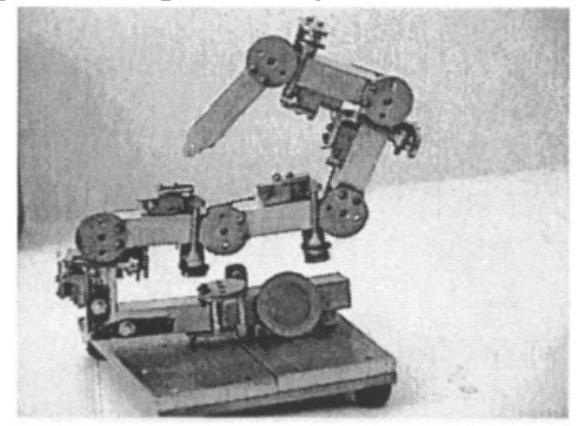

Figure 2. Sequential micromanipulator prototype

\subsection{Computer vision system}

To obtain a low cost microequipment it is necessary to use low cost components. Low cost components do not permit us to obtain high absolute accuracy of the assembly devices. To avoid this drawback we have developed an adaptive algorithm for microassembly using a technical vision system (Fig. 3).

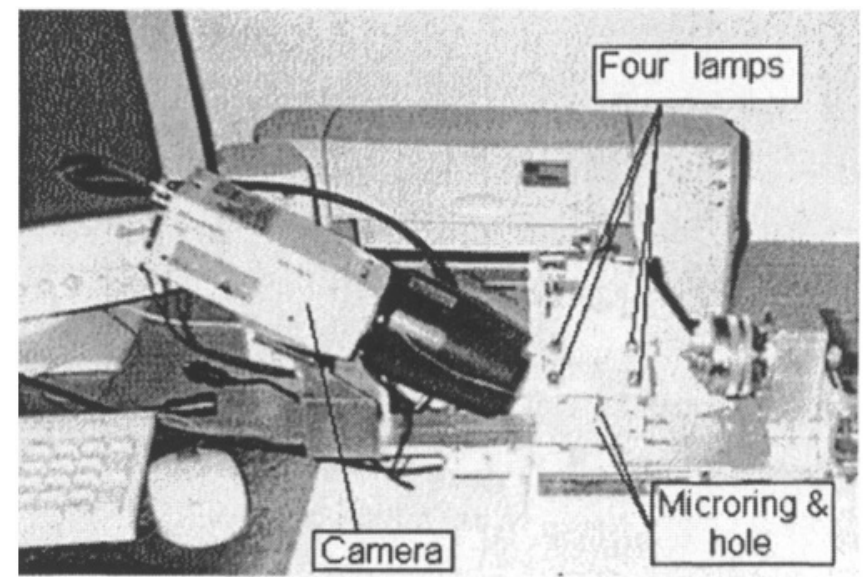

Figure 3. The prototype of visual controlled assembly system

The main idea of this approach is to replace the stereovision system, which demands two video cameras, for the system with one TV camera for teleconferences, with a cost of 40 dollars, and four light sources. The shadows from the light sources permit us to obtain the 3-D position of the 
needle with the microring relative to the hole. The microring is to be inserted into the hole. We use a neural classifier to recognize the relative position.

The problem of automatic microdevices assembly is very important in mechatronics and micromechanics. To obtain the high precision, it is necessary to use adaptive algorithms on the base of technical vision systems. We proposed an approach, that permits us to develop the adaptive algorithms based on neural networks. We consider the conventional pin-hole task. It is necessary to insert the pin into the hole using a low cost technical vision system.

For this purpose it is necessary to know the displacements $(d x, d y, d z)$ of the pin tip relative to the hole. It is possible to evaluate these displacements with a stereovision system, which resolves $3 \mathrm{D}$ problems. The stereovision system demands two TV cameras. To simplify the control system we propose the transformation of 3D into 2D images preserving all the information about mutual location of the pin and the hole. This approach makes it possible to use only one TV camera.

Four light sources are used to obtain pin shadows. Mutual location of these shadows and the hole contains all the information about the displacements of the pin relative to the hole. The displacements in the horizontal plane $(d x, d y)$ could be obtained directly by displacements of shadows center points relative to the hole center. Vertical displacement of the pin may be obtained from the distance between the shadows. To calculate the displacements it is necessary to have all the shadows in one image. We capture four images corresponding to each light source sequentially, and then we extract contours and superpose four contour images. We use the resulting image to recognize the position of the pin relative to the hole. We developed the neural network system which permits us to recognize the pin-hole displacements with errors less than 1 pixel $[11,12]$.

\subsection{Adaptive Algorithm of the Lowest Level}

To compensate for the machine tool errors we have developed a special algorithm for the workpiece diameter measurement using the electrical contact of the workpiece with the measurement disk (Fig. 4). This measurement allows us to develop the algorithm for brass needle cutting. We obtained a brass needle with a diameter of $50 \mu \mathrm{m}$ and a length of $550 \mu \mathrm{m}$ (Fig. 5) almost equal to the Japanese needle [18]. 


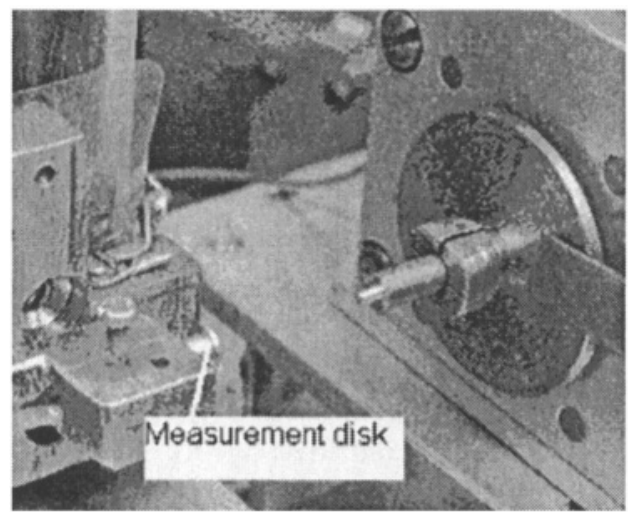

Figure 4. The workpiece with measurement disk

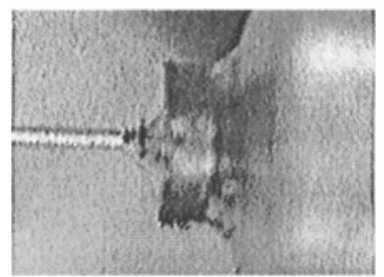

Figure 5. The brass needle with $\mathbf{5 0} \boldsymbol{\mu m}$ diameter

\subsection{Genetic Algorithm for Micromachine Tool Characterization}

To improve the micromachine tool precision it is necessary to correct its errors. To obtain the information about the micromachine tools errors, we use a two balls scheme for machine tool parameters measurement. One ball is fixed on the special tool support, which is inserted to the chuck. The second ball is fixed on the machine tool carriage (Fig. 6).

By moving the carriage with the second ball up to the contact with the first ball in different positions it is possible to obtain all the needed information about the geometrical properties of the machine tool. But the geometrical parameters depend on the contact positions in a very complicated manner. To resolve the system of nonlinear equations which represent the mentioned dependence we use a genetic algorithm. This approach permits us to reduce to one third the micromachine tools errors. 


\section{CONCLUSIONS}

AI algorithms could be used to increase the level of manufacturing processes automatization. The experiments with AI algorithms in real industry factories are too expensive. In this article a low cost test bed for AI method examinations is proposed. This test bed is composed of the micromechanical models of conventional industry devices. The prototypes of micromachine tools and micromanipulators were developed and examined with some AI algorithms. The test bed examination results show that AI systems could be proved with low expenses.

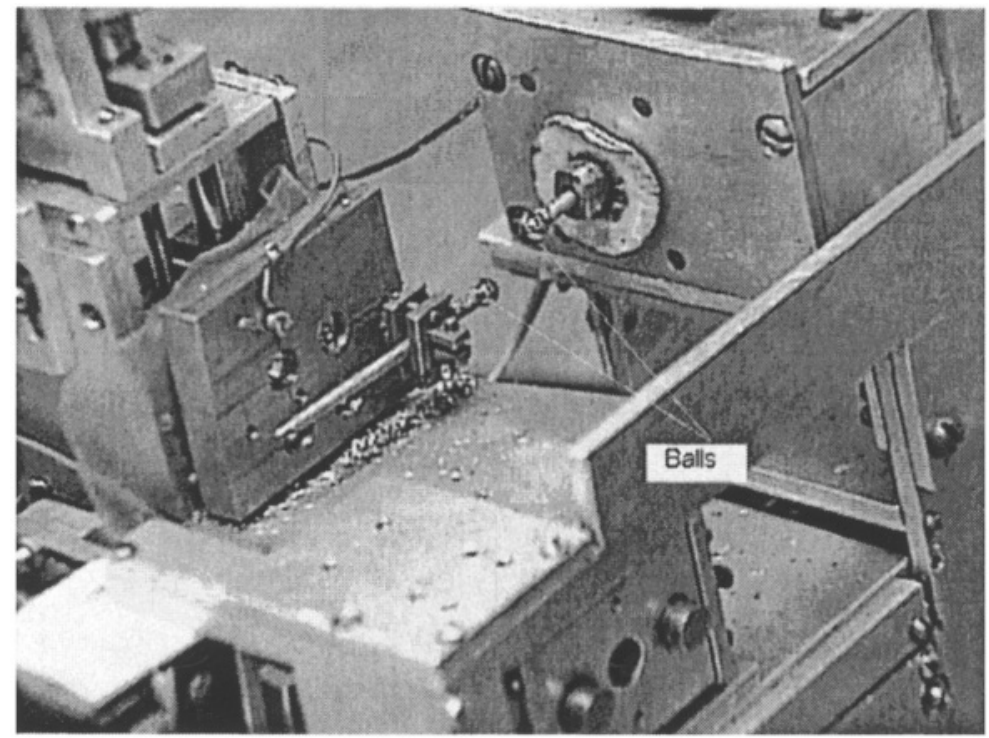

Figure 6. Ball location in the micromachine tool

\section{ACKNOWLEDGEMENTS}

This work was supported by CCADET, UNAM, projects CONACYT 33944-U, PAPIIT 1112102, NSF-CONACYT 39395-A.

\section{REFERENCES}

1. Eberhart, R.: Overview of computational intelligence [and biomedical engineering applications]. Proceedings od the 20-th Annual International Conference of the IEEE Engineering in Medicine and Biology Society 3 (1998) 1125-1129 
2. Hui, T., Brown, D., Haynes, B., Xinxian Wang: Embedded e-diagnostic for distributed industrial machinery. IEEE International Symposium on Computational Intelligence for Measurement Systems and Applications (2003) 156-161

3. Awadallah, M., Morcos, M.: Application of AI tools in fault diagnosis of electrical machines and drives-an overview. IEEE Transactions on Energy Conversion 18, Issue 2 (2003) 245-251

4. Werbos, P.: Advanced Forecasting Methods for Global Crisis Warning and Models of Intelligence. In: General Systems Yearbook 22 (1977) 25-38

5. Prokhorov, D., Wunsch, D.: Adaptive Critic Designs. IEEE Trans. on Neural Networks 8, N 5 (1997) 997-1007

6. Xiaoou Li, Lara-Rosano, F.: A weighted fuzzy Petri net model for knowledge learning and reasoning. International Joint Conference on Neural Networks, IJCNN '99 42368 -2372

7. Xiaoou Li, Wen Yu, Lara-Rosano, F.: Dynamic Knowledge Inference and Learning under Adaptive Fuzzy Petri Net Framework. IEEE Transactions on Systems, Man, and Cybernetics 39, N4 (2000) 442-450

8. Bottou, L., Cortes, C., Denker, J., Drucker, H., Guyon L., Jackel L., LeCun J., Muller U., Sackinger E., Simard P., Vapnik V.: Comparison of Classifier Methods: a Case Study in Handwritten Digit Recognition. In: Proceedings of $12^{\text {th }}$ IAPR International Conference on Pattern Recognition 2 (1994) 77-82

9. Fukushima, K. Neocognitron: A hierarchical neural network capable of visual pattern recognition. Neural Networks 1 (1988) 119-130

10. Roska, T., Rodriguez-Vazquez, A.: Toward visual microprocessors. Proceedings of the IEEE 90, Issue 7 (July 2002) 1244-1257

11. Baidyk, T.: Application of Flat Image Recognition Technique for Automation of Micro Device Production. Proceedings of the International Conference on Advanced Intelligent Mechatronics “AIM'01”, Italy (2001) 488-494

12. Baidyk, T., Kussul, E., Makeyev, O., Caballero, A., Ruiz, L., Carrera, G., Velasco, G.: Flat image recognition in the process of microdevice assembly. Pattern Recognition Letters 25/1 (2004) 107-118

13. Kussul, E.: Micromechanics and the perspectives of neurocomputing. In: Neuron-like Networks and Neurocomputers, Kiev, Ukraine (1993) 76-82 (in Russian)

14. Kussul, E., Rachkovskij, D., Baidyk, T., Talayev, S.: Micromechanical Engineering: a Basis for the Low-cost Manufacturing of Mechanical Microdevices Using Microequipment. Journal of Micromechanics and Microengineering 6 (1996) 410-425

15. Kussul, E., Baidyk, T., Ruiz-Huerta, L., Caballero, A., Velasco, G., Kasatkina, L.: Development of Micromachine Tool Prototypes for Microfactories. Journal of Micromechanics and Microengineering 12 (2002) 795-812

16. Wenxin Liu, Venayagamoorthy, G., Wunsch, D.: A heuristic dynamic programming based power system stabilizer for a turbogenerator in a single machine power system. $39^{\text {th }}$ IAS Annual Meeting on Industry Applications 1 (2003) 270-276

17. Croce, F., Delfino, B., et al.: Operations and management of the electric system for industrial plants: an expert system prototype for load-shedding operator assistance. IEEE Transactions on Industry Applications 37, Issue 3 (2001) 701-708

18. Okazaki, Yu., Kitahara T.: Micro-Lathe Equipped with Closed-Loop Numerical Control. Proceedings of the 2-nd International Workshop on Microfactories, Switzerland (2000) 8790 